\section{Gebäudekrankheit: Symptome fast immer psychisch bedingt}

Konkrete physikalische oder chemische Ursachen für das Sick Building Syndrome (SBS) lassen sich nach wie vor nicht ausmachen. Auch eine neue, breit angelegte Studie kommt zu dem Ergebnis, daß es überwiegend psychosoziale Faktoren sind, die bei den Patienten am Arbeitsplatz körperliche Symptome auslösen. Lediglich bei einer schlechten Belüftung kann evtl. das Gebäude für die Beschwerden verantwortlich gemacht werden.
Das Sick Building Syndrome (SBS) ist seit den 80er Jahren auch in breiten Be„Gebäudekrankheit" bekannt und umschreibt unspezifische, mit Gebäuden zusammenhängende Beschwerden. Die Patienten klagen über trockene Schleimhaut von Mund und Nase, Kopfschmerzen, Müdigkeit und Konzentrationsstörungen sobald sie sich in einem bestimmten Gebäude - meist ihrem Arbeitsplatz - befinden.

Umweltmediziner haben sich dieses Problems angenommen und versucht, die Symptome auf bestimmte physikalische oder chemische Determinanten zu reduzieren: Sind durch die Klimaanlage verbreitete Schimmelpilzsporen, Formaldehyd aus den Büromöbeln, Ozon von Kopierer und Laserdrucker oder einfach die Luftfeuchtigkeit schuld am SBS?

Bislang sind diese Untersuchungen allerdings nicht von Erfolg gekrönt zu sehr spielen psychologische Faktoren wie Zufriedenheit mit der Arbeit oder allgemeines Streßniveau eine statistisch signifikante Rolle und sind daher nicht zu vernachlässigen. Dies belegt erneut eine auf der diesjährigen International Multidisciplinary Conference on Environmental Medicine in Graz vorgestellte Multizenterstudie.

In dieser Untersuchung wurden über 4500 Arbeitnehmer in 14 Bürogebäuden über mögliche SBS-assoziierte völkerungskreisen unter dem Namen
Beschwerden befragt. Gleichzeitig untersuchte man die Probanden medizinisch-psychologisch und erfaßte Daten über das Innenraumklima.

Ergebnis: Sensorische Beschwerden als Indikator für ein SBS waren bei Arbeitnehmern in dem gesamten $\mathrm{Ge}$ bäude anzutreffen und betrafen kein einzelnes Büro oder eine einzelne Etage. Eine starke statistische Korrelation bestand zwischen einer allgemein ein- geschränkten Gesundheit und psychosozialen Faktoren wie Arbeitsunzufriedenheit, nicht zu schaffendem Arbeitspensum oder $\mathrm{zu}$ hohem oder dauerndem Arbeitsstreß. Die Betroffenen schätzten auch das Innenraumklima subjektiv als schlecht ein.

Ein objektives Kriterium konnte dennoch festgestellt werden: Sind die Büroräume gut belüftet, klagen weniger Probanden über SBS als wenn die Räume schlecht belüftet sind. Der Frage aber, ob es in den schlecht belüfteten Büroräumen zu einer Anhäufung von Schadstoffen kommt, wurde in der Untersuchung nicht nachgegangen.

Fazit: Den Patienten, die über SBS klagen, kann weiterhin kaum etwas Tröstliches gesagt werden. Zumindest erscheint es bei der jetzigen Datenlage nicht zwingend notwendig, eine große Umwelt- oder Allergiediagnostik einzuleiten. Ein Hinweis auf die Belüftungsverhältnisse kann im Einzelfall helfen. Im Einzelfall muß auch entschieden werden, ob ein ausführliches Gespräch über die Arbeitszufriedenheit zu einem Rückgang der Symptome führen könnte.

S. von Mackensen, M. Bullinger, M. Morfeld, München, anläßlich der International Multidisciplinary Conference on Environmental Medicine, Graz 1999.

\section{Innenraumklima in Büros: Das Märchen von der Ozonbelastung}

Büroangestellte beklagen sich häufiger über die „Ozonbelastung“ in Innenräumen, die vor allem in der kalten Jahreszeit nicht gut gelüftet werden. Das „Ozon“ führt bei ihnen zu Kopfschmerzen oder Irritationen der Schleimhäute.

Fest steht, daß Geräte, die mit UV-Strahlung arbeiten - dazu gehören Kopierer und Laserdrucker, aber auch elektrisch arbeitende Raumluftreiniger -, Ozon emittieren können. Dies gilt aber eher für die älteren Geräte, neue sind mit einem entsprechenden Filtersystem ausgestattet. Eine Vor-Ort-Untersuchung von Mitarbeitern des Lehrstuhls für Bioklimatologie und Immissionsforschung der Universität München, durchgeführt in normalen Büroräumen mit Kopierern und Laserdruckern wie auch in einem Copyshop, bestätigen das. Die dort gemessenen Ozonwerte bewegten sich teilweise hart an der Nachweisgrenze. Dies galt auch für Räume, in denen Luftreinigungsgeräte betrieben wurden. Und selbst wenn ältere Geräte Ozon produzieren sollten das chemisch sehr instabile Molekül hat in Innenräumen eine Halbwertszeit von nur wenigen Minuten.

G. Jakobi, P. Fabian, München, anläßlich der International Multidisciplinary Conference on Environmental Medicine, Graz 1999 